\title{
Psychometric Properties of the Bengali Version Brief Questionnaire on Smoking Urges (QSU-Brief)
}

\author{
Shakil Ahmed ${ }^{1 *}$, Fabeha Tazmeem', Mohammad Morshad Alam², Md Golam \\ Kibria ${ }^{3}$, Badrut Tamam Ahmed', Bappi Bhushan Das', Abdullah Al Amin ${ }^{1}$, \\ Ahmed Hossain ${ }^{1}$
}

\begin{abstract}
The study aimed to investigate the factorial structure, reliability, and validity of the Bengali version of the brief Questionnaire on Smoking Urges (QSU-Brief) tool in a sample of Bangladeshi smokers. The Bengali version QSU-Brief scale's reliability and validity were assessed on the basis of the data provided by 460 Bangladeshi smokers. To substantiate the data reliability, internal consistency and test-retest reliability were measured. Exploratory factor analysis (EFA), followed by confirmatory factor analysis (CFA), was conducted to validate the psychometric properties of the 10-item-QSU-Brief tool. The questionnaire showed good internal consistency (Cronbach's $\alpha=0.94$; Intraclass correlation coefficient $=0.91 ; \mathrm{p}<0.001)$. The EFA and CFA confirmed that a two-factor solution explained $75.1 \%$ of the total variance and considered the best item structure of the Bengali version of QSU-Brief across the current study setting. The first factor reflected a strong desire to smoke, which comprised items $1,2,3,5,6,9$, and 10. While the second factor displayed expectation of relief from the negative implications, which contained items 4,7 , and 8 . The study findings showed that the Bengali QSU-Brief had good reliability, validity, and factorial structure. Therefore, this tool could be an excellent candidate to evaluate smoking urges in Bangladeshi settings.
\end{abstract}

Keywords: Validity and reliability- QSU-Brief- smoking- factor structure- Bangladesh

Asian Pac J Cancer Prev, 22 (12), 3857-3863

\section{Introduction}

More than a billion people worldwide smokes which majority are from low- and middle-income countries (World Health Organization, 2019). Bangladesh is one of the developing countries with high smoking prevalence rate and is in increasing rate. Around $36 \%$ of men and $1 \%$ of female here smoke either cigarettes or bidis (Burki, 2019; Ritthiphakdee, 2019). Bangladesh is also one of the 15 countries with a greater burden of tobacco-associated illness and smoking is behind $25 \%$ of death in (Hakim et al., 2018; Sultana et al., 2018).

The WHO and UNDCP jointly defined "Craving Mechanism" as the concept behind smoking addiction (UNDCP and World Health Organization, 1992). This is corelated with various factors and hampers the success smoking cessation programs (Littel et al., 2011). The measurement of 'craving' is important to determine in cessation programs for a number of reasons. For example, overall nature, testing intervention, forecast relapse, and overall treatment outcome (West and Ussher, 2010).

The tools that were used to assess 'craving' previously had limitation regarding psychometric properties, reliability, and limited number of questions in assessment tolls (Little et al., 2011). To overcome these limitations, in 1991 Tiffany and Drobes developed the Questionnaire on Smoking Urges (QSU) consisting 32 items. This is a self-report instrument able to determine reliable and psychometrically toned 'craving' from various aspects (Tiffany and Drobes, 1991).

Originally, QSU represented four different types of smoking urges includes desire, expectancy of early positive result, expectation of early relief and intention to smoke (Tiffany and Drobes, 1991). Though early psychometric assessment verified the use of QSU for 'craving' measurement, however recently researcher have reported inconveniency of this tool (Toll et al., 2006). Moreover, it is less appropriate in laboratory settings and where immediate assessment is required due to its length. Previous study also suggests that, a fewer item would make the scale more psychometrically sound (Little et al., 2011; West and Ussher, 2010). Indeed, A QSU-Brief was developed by Cox et al., (2001) to address those limitations. This is an abbreviated form of initial QSU tool consist of ten items and usable in settings where immediate assessment is required and developed to render

${ }^{1}$ Department of Public Health, North South University, Dhaka-1229, Bangladesh. ${ }^{2}$ Health, Nutrition, and Population Global Practice, The World Bank, Dhaka-1207, Bangladesh. ${ }^{3}$ Centre for Development Action, Dhaka, Bangladesh. *For Correspondence: shakil.ahmed3@northsouth.edu. Shakil Ahmed and Ahmed Hossain have equal contribution in this study. 
the two factor structure of the first model. The QSU-Brief scale also has sound reliability $(\alpha=0.78-0.97)$ which is comparable to original QSU. The psychometric properties have also been confirmed by various research articles (Cappelleri et al., 2007; Little et al., 2011). Afterwards, the QSU-Brief has been successfully used in a number of researches worldwide (Blebil et al., 2015; Littel et al., 2011).

Bengali is the $5^{\text {th }}$ largest language in the world with around 228 million native speakers and another 37 million as second language (Ethnologue, 2019). Unfortunately, till now no smoking 'craving' tools has been validated for Bengali linguists' people. Due to lacking of validated tools, research in this area is very limited among Bengali people. In clinical settings, they might be relied on a non-validated translation. Therefore, we have taken the decision of translating QSU-Brief tool to evaluate smoking craving in Bengali language, to carry out research in both the clinical and non-clinical settings. Our research aimed to focus on translating the QSU-Brief tool, assessing its factorial structure, reliability and validity of the questionnaire for future potential use.

\section{Materials and Methods}

\section{Participants}

This current study adopted a cross-sectional design to evaluate the data from the smokers of Bangladesh, enrolled in a mixed-method research identifying the risk factors of being non-compliant towards smoke-free policy issued by the Bangladesh government. Participants were 460 adults ( $\geq 18$ years) male, smoked at least 100 cigarettes in their lifetime, and had no current plan to refrain from smoking. Eligible participants were public transport (i.e., bus and human hauler) drivers, who drove the vehicles inside Dhaka city and had a valid professional driving license issued by the Bangladesh Road Transport Authority (BRTA) (Bangladesh Road Transport Authority, 2020).

\section{Measures}

A semi-structured questionnaire was used, comprised of several sections of questions, including demographic and rating scales, to obtain necessary data from the study participants. First, participants were asked about their demographic (age, marital status, education, etcetera) and relevant smoking characteristics (age of starting their smoking habit, number of cigarettes smoked per day, duration of smoking, etcetera). The participants were then asked to answer the brief version Bengali translation of the Questionnaire on Smoking Urges (QSU-Brief) scale, which measures the smoking urges and cravings (Cox et at., 2001). The QSU-Brief is a 7-item Likert-type response scale ranging from 1 (strongly disagree) to 7 (strongly agree) and comprised of 10 questions (Table 1: supplementary file 1). The standard guidelines were followed during the Bengali translation of the QSU-Brief tool (Wild et al., 2005). One Bengali linguists translated the Bengali version of the QSU-Brief. During the translation, a native English speaker guided the linguist, and discussion continued until both parties achieved unanimity. After that, the piloting of the translated questionnaire among 25 native Bengali speakers was carried out. Each of the pilot testing participants was interviewed by a counselor to receive the review and feedback on the translated version. The researchers examined the reviews and feedbacks. By addressing the relevant and required changes from the reviews and feedback, the final edition of the Bengali translated 10-items-QSU-Brief was achieved.

Furthermore, all participants were questioned on the Bengali translated Fagerstrom Test for Nicotine Dependence scale (FTND) (Mushtaq et al., 2019), which had 6 questions assessing the smoking habit and the nicotine dependence of the study participants (Cronbach's alpha $=0.7)$. The participants also completed the Modified Reasons for Smoking Scale (MRSS) (Berlin et al., 2003), and this tool had 21 questions. The answers were given on a 5-item Likert type responses scale from 1 (never) to 5 (always). The MRSS tool was used to evaluate the reasons for smoking in a detailed psychological profile among smokers (Cronbach's alpha $=0.8$ ).

\section{Statistical procedure}

The demographic characteristics and relevant smoking indicators were summarized by descriptive statistics. The mean and standard deviation were analyzed for continuous variables. The frequency and percentages were determined for categorical variables. The normality of the continuous variables was checked using Shapiro-Wilk's test. The Cronbach's alpha coefficient and test-retest reliability were measured to assess the internal consistency and homogeneity for both the Bengali translated 10-itemsQSU-Brief tool and its estimated factors. For confirming the suitability of the dataset to carry out factor analysis, the Kaiser-Meyer-Olkin (KMO) test and Bartlett's test of sphericity were applied. The validation of the psychometric properties of the tool was measured by employing factor analysis and concurrent validity. Exploratory factor analysis (EFA) was carried out to elucidate the Bengali version QSU-Brief items' factorial structure. The native inter-item correlation structure was conserved by applying the promax rotation method. A scree plot was illustrated to establish the optimum numbers of factors in the EFA solution. The number of items within a factor was selected if the item's factor loading value $\geq 0.40$. After the EFA, a confirmatory factor analysis (CFA) was performed. The overall chi-squared fit index value, comparative fit index (CFI), root mean square error of approximation (RMSEA), Tucker-Lewis index (TLI) was estimated. For the RMSEA, a value $<0.08$ considered acceptable, and a value $>0.10$ advanced to rejection of the model; for the TLI, a value $>0.9$ was considered a good fit. A loading value $>0.50$ for standardized factor loadings was considered a good fit, and a value $>0.40$ implied an acceptable correlation. Any loading value below 0.40 indicated a low correlation. The concurrent validity of the tool was measured by establishing the correlation between the Fagerstrom test for nicotine dependence (FTND), modified reasons for smoking scale-21 (MRSS-21), number of cigarettes smoked per day, and duration of smoking (in years). 
This validation was evaluated by utilizing the Pearson Correlation Coefficient test. The construct validity was determined by confirming the desired correlation and the validated measure's outcome (Miller and Lovler, 2019). All the analyses were performed using R (v. 4.0.2)(R Core Team, 2020), and "tidyverse" (Wickham and RStudio, 2019), "psych”(Revelle, 2017), "GPArotation"(Bernaards and Jennrich, 2015), "lavaan"(Rossel et al., 2020), and "PerformanceAnalytics"(Peterson et al., 2020) packages were utilized.

\section{Results}

Participant characteristics and relevant smoking information are shown in Table 1. Out of 460 smoker participants, 406 were Bus driver and 54 were human hauler drivers. All the participants were male. The mean age of our study participants was 30.59 years, with a standard deviation of 7.88 years. The majority (79.1\%) belonged to the ever-married group. The mean education of our study participants was estimated to be 5.95 years $(\mathrm{SD}=3.31)$.

\section{Exploratory factor analysis}

The overall Kaiser-Meyer-Olkin measure for the Bengali translation of the QSU-Brief scale was 0.94, indicating that the sample size was acceptable for exploratory factor analysis (EFA). Besides, Bartlett's test of sphericity also exhibited the dataset's suitability $(\mathrm{p}<0.001)$.

EFA was employed to all the items of QSU-Brief with the promax rotation for the validation of the
Psychometric Properties of the Bengali Version QSU-Brief Scale

Table 1. Characteristics of the Study Respondents $(\mathrm{N}=460)$

\begin{tabular}{lc}
\hline Variables & Mean \pm SD \\
\hline Age (years) (Mean \pm SD) & $30.59 \pm 7.88$ \\
Vehicle Types, n (\%) & \\
Bus & $406(88.3)$ \\
Human Hauler & $54(11.7)$ \\
Marital Status, n $(\%)$ & \\
Ever Married & $364(79.1)$ \\
Never Married & $96(20.9)$ \\
Education in years $(M e a n \pm$ SD) & $5.95 \pm 3.31$ \\
Age of starting smoking (Mean \pm SD) & $16.12 \pm 4.3$ \\
Number of cigarettes smoked/day $(M e a n \pm S D)$ & $12.58 \pm 5.99$ \\
Duration of smoking (years) $(M e a n \pm$ SD) & $14.47 \pm 7.89$ \\
FTND total score (Mean \pm SD) & $2.72 \pm 1.83$ \\
MRSS-21 & $44.34 \pm 10.93$ \\
\hline
\end{tabular}

questionnaire's structure. The promax rotation is supposed to increase the scientific acceptability and interpretability of the Bengali version QSU-Brief tool's factorial structure. The EFA, revealed a two factors solution with $75.1 \%$ cumulative variance.

To establish a good simple factorial structure, the loadings $\geq 0.40$ were considered for every item. Therefore, it appears the factor 1 reflected seven items (items 1, 2, 3, $5,6,9$, and 10), and factor 2 reflected three items (items 4,7 , and 8). The factor 1 displayed a strong craving for smoking and an expectation of relief from the pessimistic influence was portrayed by the factor 2 . All the items with

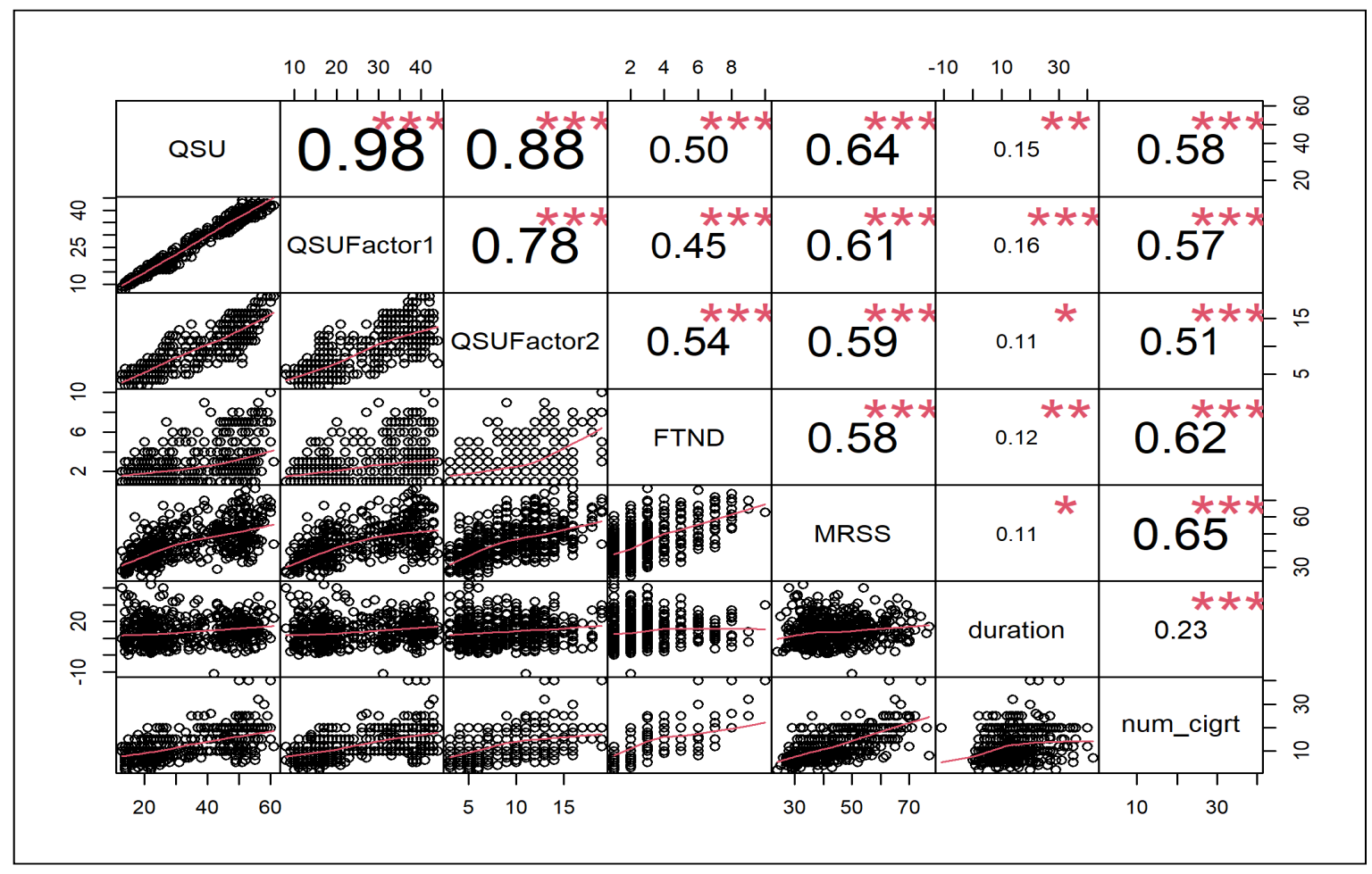

Figure 1. Correlation Matrix between QSU-Brief and Smoking Related Variables. Note: Pearson correlation method was applied as normality of the variables were obtained. Abbreviation: QSU (Questionnaire on smoking urges - Brief); QSUFactor1 (QSU - Brief Factor 1: reflecting a strong desire to smoke); QSUFactor2 (QSU-Brief Factor 2: presented the expectation to get relief from negative effect), FTND (Fagerstrom Test for Nicotine Dependence); MRSS (Modified reasons for smoking scale); duration (Duration of smoking); num_cigrt (Number of cigarettes smoked per day) 
Table 2. Factor Structure of Brief Questionnaire of Smoking Urges Items $(\mathrm{N}=460)$

\begin{tabular}{llcl}
\hline Items & & Factor 1 & Factor 2 \\
\hline 1. & I have a desire for a cigarette right now. & 0.828 \\
2. & Nothing would be better than smoking a cigarette right now. & 0.567 \\
3. & If it were possible, I probably would smoke right now. & 0.818 & 0.759 \\
4. & I could control things better right now if I could smoke. & 0.674 & 0.773 \\
5. & All I want right now is a cigarette. & & 0.616 \\
6. & I have an urge for a cigarette. & 0.416 & 0.686 \\
7. & A cigarette would taste good now. & 0.937 & \\
8. & I would do almost anything for a cigarette now. & & \\
10. & Smoking would make me less depressed & I am going to smoke as soon as possible. & \\
\hline
\end{tabular}

Note: Promax rotation method was used. Only loadings $>0.40$ are shown

their loading value are shown in Table 2.

\section{Confirmatory factor analysis}

The two-factor solution of the Bengali translation of the QSU-Brief tool achieved from the EFA was tested and confirmed by confirmatory factor analysis (CFA). The CFA exhibited an acceptable model fit $\left(\chi^{2}=182.7\right.$; $\mathrm{CFI}=0.96$; $\mathrm{TLI}=0.95 ; \mathrm{RMSEA}=0.077)$.

Table 3 demonstrates the Bengali version QSU-Brief tool's standardized factor loadings, and all the loadings of this model were found statistically significant $(p<0.001)$. All the items' loadings of this model except one (item 9) were greater than 0.50 , which indicates a good fit. However, the loading value of item 9 was 0.48 , which implied to be an acceptable correlation.

\section{Reliability of the Bengali version QSU-Brief questionnaire}

Cronbach's alpha coefficient value was estimated to be 0.94 (Table 3). Hence, it could be hypothesized that the Bengali version QSU-Brief scale has adequate internal consistency (Taber, 2018). The intraclass correlation coefficient (ICC) for the QSU-Brief scale was measured to be 0.91 (Table 3). Thus the ICC presented excellent reliability for the Bengali translation of this QSU-Brief scale (Koo and Li, 2016).
The Cronbach's alpha score was also good for the separate factors, i.e. Factor 1 (Items 1, 2, 3, 5, 6, 9, and $10 ; \alpha=0.93$ ) and Factor 2 (Items 4, 7, and 8; $\alpha=0.77$ ). Similarly, the ICC score was good as for both the factor $1(\mathrm{ICC}=0.92 ; \mathrm{p}<0.001)$ and factor $2(\mathrm{ICC}=0.63$; $\mathrm{p}<0.001)$ (Table 3).

\section{Concurrent validity}

The data normality was checked by the Shapiro-Wilk test. The test statistics for the potential variables are as follows: overall score of Bengali translated 10-items-QSU-Brief tool ( $\mathrm{w}=0.91 ; \mathrm{p}<0.01)$, FTND total score $(\mathrm{w}=0.82 ; \mathrm{p}<0.001)$, MRSS-21 total score $(\mathrm{w}=0.96 ; \mathrm{p}<0.001)$, duration of smoking in years $(\mathrm{w}=$ $0.97 ; \mathrm{p}<0.001$ ), and number of cigarettes smoked per day $(\mathrm{w}=0.91 ; \mathrm{p}<0.001)$ of the participants. Therefore, the normality for each of the variables was obtained. The Figure 1 illustrates the correlation matrix measured by Pearson correlation coefficients between the total score of the QSU-Brief tool and other relevant smoking characteristics. It appears from the figure- 2 that, the overall score of Bengali version 10-items- QSU-Brief had high and significant positive correlation with Factor $1(\mathrm{r}=0.98 ; \mathrm{p}<0.001)$ and Factor $2(\mathrm{r}=0.88 ; \mathrm{p}<0.001)$ of the QSU-Brief, FTND's overall score $(r=0.50 ; p<0.001)$,

Table 3. Results of Confirmatory Factor Analysis (Standardized Factor Loadings), Cronbach's Alpha, Test Retest Reliability (Intraclass Correlation Coefficient) of the QSU-Brief $(\mathrm{N}=460)$

\begin{tabular}{llcc}
\hline Items & & Factor 1 & Factor 2 \\
\hline 1. & I have a desire for a cigarette right now. & 0.95 & 0.83 \\
2. & Nothing would be better than smoking a cigarette right now. & 0.94 & 0.77 \\
3. & If it were possible, I probably would smoke right now. & & 0.92 \\
4. & I could control things better right now if I could smoke. & 0.94 & 0.91 \\
5. & All I want right now is a cigarette. & & 0.52 \\
6. & I have an urge for a cigarette. & & 0.48 \\
7. & A cigarette would taste good now. & 0.67 & 0.77 \\
8. & I would do almost anything for a cigarette now. & 0.93 & $0.63^{* * *}$ \\
9. & Smoking would make me less depressed & $0.92^{* * *}$ & \\
Cronbach's Alpha (For the questionnaire $=0.94)$ & & \\
Intraclass correlation coefficient (For the questionnaire $\left.=0.91^{* * *}\right)$ & & \\
\hline
\end{tabular}

*p $<0.05 ; * * \mathrm{p}<0.01 ; * * \mathrm{p}<0.001$ (two tailed) 
MRSS-21's overall score $(r=0.64 ; \mathrm{p}<0.001)$, and number of cigarettes smoked per day $(r=0.58 ; \mathrm{p}<0.001)$. Only duration of smoking in years $(r=0.15 ; \mathrm{p}<0.01)$ indicators showed a low degree correlation.

\section{Discussion}

The current study evaluated the psychometric properties of the Bengali translated QSU-Brief tool in the setting of Bangladesh. To our knowledge, this is the very first study that translated and assessed reliability, constructs validity, concurrent validity, and factorial structure into the Bengali language. The study's findings suggested that this Bengali QSU-Brief had shown adequate reliability and acceptable validity to determine the smoking urges among Bengali speakers of Bangladesh.

The EFA and CFA indicated a two-factor solution to this QSU-brief questionnaire explained the data optimally. This two-factor solution finding endorsed the findings of the English, Malay, and French version of the QSU-Brief (Blebil et al., 2015; Clausius et al., 2012; Cox et al., 2001; Dethier et al., 2014). However, in 1996, Kozlowski et al. suggested a single factor solution might also be a better fit for the QSU-Brief questionnaire (Kozlowski et al., 1996).

In this current study, the first factor of Bengali QSU-Brief consisted of 7-items (i.e., items 1, 2, 3, 5, 6, 9, 10); followed by the second factor that included the rest of 3-items (i.e., 4, 7, and 8). However, in the 10-item English QSU-Brief two factor solution from Cox et al., showed factor 1 was comprised of 5 items, i.e., item no. 1, 3, 6, 7, 10 , and factor 2 contained item no. 2, 4, 5, 8, and 9 (Cox et al., 2001). The Malay QSU-Brief also showed a factorial structure similar to the model of Cox et al. (Blebil et al., 2015; Cox et al., 2001). This dissimilarity might occur due to cultural and language variation. However, the first factor from the current study displayed a strong desire or intention to smoke, and the second factor showed an expectation of relief from negative implications, which comes with an agreement to both English and Malay version of 10-item QSU-Brief (Blebil et al., 2015; Cox et al., 2001; Toll et al., 2006).

The exploratory factor analysis indicated a strong loading value in the items 1,3 and 10 from factor 1 . While, Blebil et al. showed a convincing loading in item 1 and 3, but dubious loading in item-10 (Blebil et al., 2015). In the Dutch QSU-Brief tool, displayed a high loading in item 3 and 10 , yet poor loading in item-1. The Dutch version QSU-Brief, contrasted the highest loading in items 2 and 5 (Little M., 2011). However, in our study findings the item 2 and 5 both loaded in the factor 1 with a mediocre loading value.

The current study showed strong significant correlations between the 10-items Bengali QSU-Brief tool's total score and the overall score of FTND, duration of smoking in years, the number of cigarettes smoked/day, and total score of 21-items modified reasons for smoking scale. Meanwhile, Blebil et al. found a significant positive correlation between the Malay QSU-Brief tool, the number of cigarettes smoked/day, and the participants' carbon monoxide (CO) level. They did not find any significant relation between smoking duration and the 10-items-QSU-
Brief tool. Besides, in our present study, no CO level had been measured, and Blebil et al., (2015) did not use the MRSS tool to confirm the concurrent validity. In the Dutch version of the QSU-Brief scale, Little et al., (2011) showed concurrent validity by estimating significant correlations between the QSU-Brief tool and FTND, cigarettes smoked/day, and some rating scales. Little et al., (2011) found strong positive significant correlations between FTND and cigarettes smoked/day with the total score of Bengali QSU-Brief tool. These findings from Franken et al. corresponded to the findings of our study. Our study utilized the MRSS tool; to validate the concurrent validity, and our literature search no other studies used this specific tool to validate the QSU-Brief tool in any language to date.

This present study had some limitations to be mentioned. No female participants could be recruited in this present study. This limitation could be explained due to the cultural pattern, context, and restriction in Bangladesh. Our current study population was public transport (i.e., buses and human haulers) drivers. Therefore, this is considered taboo for a woman to drive either a bus or a human hauler in the urban city area (The Financial Express, 2020). Thus, no eligible women candidate could be found in this current study from the public transport driver's community. Besides, the prevalence of female smoker $(1.5 \%)$ in Bangladesh is considerably lower compared to their male $(44.7 \%)$ counterparts (World Health Organization, 2017). An additional limitation was, in our study, we did not collect any information on the participants' quit attempts previously; and whether they had any mental disorder; hence the generalizability of the study findings reduced slightly. Finally, most of the studies that evaluated the psychometric properties of the QSU-Brief questionnaire had been used in clinical settings (Blebil et al., 2015; Cox et al., 2001; Toll et al., 2014). Whereas, our study had implemented the QSU-Brief questionnaire in a non-clinical setting and in a mainstream group of the population from society. Although, Cox et al. suggested that this Brief version of the QSU could be implacable as a common tool for measuring smoke cravings (Cox et al., 2001)

As a whole, the Bengali version of the 10-item QSUBrief exhibited reliability, validity, and factorial structure to measure the smoking urges in a broader community and appropriate to test in a non-clinical setting. Regardless of the good and acceptable structure of the Bengali QSU-Brief, more future research is required to endorse the factorial solutions of the current study findings in Bangladesh.

\section{Abbreviation/Acronyms:}

QSU-Brief: Questionnaire on Smoking Urges-Brief

EFA: Exploratory factor analysis

CFA: Confirmatory factor analysis

KMO: Kaiser-Meyer-Olkin

CFI: Comparative fit index

RMSEA: Root mean square error of approximation

TLI: Tucker-Lewis index

FTND: Fagerstrom test for Nicotine Dependence

MRSS-21: Modified Reasons for Smoking Scale-21 SD: Standard deviation

Asian Pacific Journal of Cancer Prevention, Vol 22 
ICC: Intraclass correlation coefficient

r: correlation coefficient

w: Test statistics of shapiro-wilk test

CO: Carbon Monoxide

Supplementary File:

Supplementary File 1: The QSU-Brief English (Supplementary Table 1) and Bengali Version (Supplementary Table 2 and 3)

\section{Author Contribution Statement}

Shakil Ahmed: Conceptualization, Methodology, Resources, Visualization, Writing - Original Draft, Supervision; Fabeha Tazmeem: Investigation, Data Curation, Writing - Original Draft; Mohammad Morshad Alam: Writing - Original Draft; Md. Golam Kibria: Writing - Review and Editing; Badrut Tamam Ahmed, Bappi Bhushan Das, Abdullah Al Amin: Field supervision; Ahmed Hossain: Conceptualization, Methodology, Supervision, Writing - Review and Editing

\section{Acknowledgments}

We are thankful to the Bangladesh Center for Communication Programs (BCCP) and Johns Hopkins Bloomberg School of Public Health, for their intense technical support to carry out the study; particularly field monitoring, data processing, and analysis. The authors also thankful to Professor Dr. Shah Monir Hossain for his technical guidance to the entire study period. We acknowledge the participants' efforts to provide their personal information for the sake of this study.

\section{Funding}

This research was supported by the $4^{\text {th }}$ Health, Population \& Nutrition Sector Program (HPNSP) of Bangladesh Medical Research Council (Grant Reference: BMRC/HPNSP-Grant/2019-2020/566).

\section{Ethical Approval}

The ethics review committee of North South University approved the study protocol as a research activity funded by Bangladesh Medical Research Council. The informed written consent was obtained from each of the study participants of this study. Besides, every participant was assured about the confidentiality of his personal information.

\section{Availability of the data}

Data can be provided on a duly justified request to the corresponding author.

\section{Conflict of Interest}

The authors report no conflicts of interest. The authors alone are responsible for the content and writing of this paper.

\section{References}

Bangladesh Road Transport Authority. (2020, February
26). Application Process of Driving License. Retrieved December 25, 2020, from http://www.brta.gov.bd/site/ page/511a7af4-20c8-4450-b4a6-5f92dde1b23f/ApplicationProcess-of-Driving-License.

Berlin I, Singleton EG, Pedarriosse AM, et al (2003). The modified reasons for smoking scale: Factorial structure, gender effects and relationship with nicotine dependence and smoking cessation in French smokers. Addict, 98, 1575-83.

Bernaards C, Jennrich R (2015). GPA Factor Rotation. Retrieved from http://www.stat.ucla.edu/research/gpa.

Blebil AQ, Sulaiman SAS, Hassali MA, et al (2015). The Malay version of the brief questionnaire on smoking urge: Translation and psychometric properties of the questionnaire. $J$ Epidemiol and Glob Health, 5, 15-22.

Burki TK (2019). Tobacco consumption in Bangladesh. Lancet Oncol, 20, 478 .

Cappelleri JC, Bushmakin AG, Baker CL, et al (2007). Multivariate framework of the Brief Questionnaire of Smoking Urges. Drug Alcohol Depend, 90, 234-42.

Clausius RL, Krebill R, Mayo MS, et al (2012). Evaluation of the brief questionnaire of smoking urges in black light smokers. Nicotine Tob Res, 14, 1110-4.

Cox LS, Tiffany ST, Christen AG (2001). Evaluation of the brief questionnaire of smoking urges (QSU-brief) in laboratory and clinical settings. Nicotine Tob Res, 3, 7-16.

Dethier V, Heeren A, Galanti L, et al (2014). Probing smoking craving with a multidimensional approach: Validation of the 12-item French-language version of the Questionnaire on Smoking Urges. Neuropsychiatr Dis Treat, 10, 1459-68.

Ethnologue. (2019). Languages of the world (22nd ed.).

Hakim S, Chowdhury MAB, Uddin MJ (2018). Correlates of attempting to quit smoking among adults in Bangladesh. Addict Behav Rep, 8, 1-7.

Koo TK, Li MY (2016). A guideline of selecting and reporting intraclass correlation coefficients for reliability research. $J$ Chiropr Med, 15, 155-63.

Kozlowski LT, Pillitteri JL, Sweeney CT, et al (1996). Asking questions about urges or cravings for cigarettes. Psych Addict Behav, 10, 248-60.

Littel M, Franken IHA, Muris P (2011). Psychometric properties of the brief Questionnaire on Smoking Urges (QSU-Brief) in a Dutch smoker population. Netherlands J Psych, 2011, $1-20$.

Miller LA, Lovler RL (2019). Foundations of Psychological Testing: A Practical Approach (6th ed.). SAGE Publications.

Mushtaq N, Huque R, Beebe LA, et al (2019). Evaluation of tobacco dependence measures in South Asian smokeless tobacco users. Drug Alcohol Depend, 203, 66-71.

Peterson BG, Carl P, Boudt K, et al (2020). Econometric tools for performance and risk analysis. R Package Version 2.0.4

R Core Team. (2020). R: A Language and Environment for Statistical Computing. Vienna, Austria: R Foundation for Statistical Computing.

Revelle W (2017). psych: Procedures for Psychological, Psychometric, and Personality Research. R Package Version 1.0-95.

Ritthiphakdee B (2019). Smoke and Mirrors in Bangladesh. Think Glob Health.

Rossel Y ves, Jorgensen TD, Rockwood N, et al (2020). Latent Variable Analysis.

Sultana P, Rahman MT, Roy DC, et al (2018). Tobacco control policies to promote awareness and smoke-free environments in residence and workplace to reduce passive tobacco smoking in Bangladesh and its correlates. PLoS One, 13, $\mathrm{e} 0198942$.

Taber KS (2018). The use of Cronbach's Alpha when developing and reporting research instruments in science education. Res 
Sci Edu, 48, 1273-96.

The Financial Express. (2020). Girl as a helper of bus. Retrieved December 27, 2020, from https://thefinancialexpress. com.bd/public/index.php/views/girl-as-a-helper-ofbus- 1514133891.

Tiffany ST, Drobes DJ.(1991). The development and initial validation of a questionnaire on smoking urges. Br J Addict, 86, 1467-76.

Toll BA, Katulak NA, McKee SA (2006). Investigating the factor structure of the Questionnaire on Smoking Urges-Brief (QSU-Brief). Addict Behav, 31, 1231-9.

UNDCP, World Health Organization (1992). Informal Expert Committee on the Craving Mechanism: Report (No. V. 92-54439T).

West R, Ussher M (2010). Is the ten-item Questionnaire of Smoking Urges (QSU-brief) more sensitive to abstinence than shorter craving measures?. Psychopharmacology, 208, 427-32.

Wickham H, RStudio (2019). Easily Install and Load the "Tidyverse."

Wild D, Grove A, Martin M, et al (2005). Principles of good practice for the translation and cultural adaptation process for patient-reported outcomes (PRO) measures: Report of the ISPOR Task Force for Translation and Cultural Adaptation. Value Health, 8, 94-04.

World Health Organization (2017). Global Adult Tobacco Survey. Retrieved from https://www.who.int/tobacco/ surveillance/survey/gats/bgd/en/.

World Health Organization. (2019). WHO Report on the Global Tobacco Epidemic, 2019. In World Health Organization (Vol. 23).

\section{c) (i) (8)}

This work is licensed under a Creative Commons AttributionNon Commercial 4.0 International License. 